\title{
Regrressão refrativa total pós-LASIK: relato de caso
}

\author{
Total refractive regression post-LASIK:case report
}

\author{
Patrícia Ioschpe Gus ${ }^{1}$ \\ Guilherme Herrmann Matos ${ }^{2}$ \\ Marcia Cristina Bayer ${ }^{3}$
}

Trabalho realizado na Disciplina de Sistema Visual da Faculdade Medicina da Universidade Luterana do Brasil.

${ }^{1}$ Professora da Disciplina de Sistema Visual da Faculdade de Medicina na Universidade Luterana do Brasil (ULBRA) Porto Alegre (RS). Doutora em Oftalmologia pela Universidade de São Paulo (USP). São Paulo (SP). Acadêmico de Medicina da ULBRA. Porto Alegre (RS). 3 Acadêmico de Medicina da ULBRA. Porto Alegre (RS).

Endereço para correspondência: Guilherme Herrmann Matos - Rua Comendador Castro, 515 - Porto Alegre (RS) CEP 91760-200

E-mail: ghmatos@globo.com ou matos81@bol.com.br

Recebido para publicação em 01.03.2004

Versão revisada recebida em 16.11.2004

Aprovação em 17.01.2005

Nota Editorial: Após concluída a análise do artigo sob sigilo editorial e com a anuência do Dr. Marcos Carvalho da Cunha sobre a divulgação de seu nome como revisor dele, agradecemos sua participação neste processo.

\section{RESUMO}

Os corticoesteróides podem aumentar a pressão intra-ocular quando administrados de maneira tópica, sistêmica e até mesmo inalatória. É rotina sua utilização após cirurgias refrativas para diminuir ou prevenir reação inflamatória. No presente relato, apresentamos o caso de uma paciente de 36 anos que, após duas semanas de cirurgia de LASIK para correção de miopia leve, teve regressão total da miopia causada pela hipertensão ocular cortisônica. O objetivo desse relato é descrever como foi conduzido o caso, as hipóteses de diagnósticos que foram levantadas, e salientar a importância da mensuração da pressão intra-ocular no pós-operatório.

Descritores: Miopia/cirurgia; corticosteróides/efeitos adversos; Ceratomileuse assistida por excimer laser in situ; Pressão intra-ocular; Glaucoma; Período pós-operatório; Relatos de casos [tipo de publicação]

\section{INTRODUÇ̃̃̃O}

Corticosteróides tópicos são rotineiramente utilizados após cirurgias refrativas para minimizar ou prevenir a reação inflamatória. Sabe-se que sua utilização, seja tópica, sistêmica ou inalatória aumenta o risco de glaucoma de ângulo aberto em um terço da população adulta, chamada de respondedora ao esteróide ${ }^{(1-2)}$. Segundo estudos mais recentes, sua administração resulta no acúmulo de glicosaminoglicanos na malha trabecular, obstruindo a drenagem do humor aquoso ${ }^{(3)}$.

O aumento da pressão intra-ocular (PIO) costuma ocorrer entre a $2^{\mathrm{a}}$ e a $6^{\mathrm{a}}$ semanas de uso do fármaco. Entretanto, isso depende das características específicas de cada droga, dose, freqüência da administração e responsividade de cada paciente. A chance de desenvolvimento de glaucoma agudo será ainda maior caso existam fatores de risco associados, tais como história familiar, idade, alta miopia, hipertensão arterial não tratada e diabetes. A descontinuidade da terapia de corticosteróide e a instituição de antiglaucomatosos geralmente normalizam a PIO em 3 a 7 dias $^{(1-3)}$.

Nesse relato, apresentamos um caso de regressão total da miopia pósLASIK em duas semanas, causada pela hipertensão ocular cortisônica. O objetivo é salientar a importância da mensuração da PIO no pós-operatório da cirurgia refrativa, e discutir os eventuais riscos do tratamento com antiinflamatório esteróide.

\section{DESCRIÇÃO DE CASO}

Paciente de 36 anos do sexo feminino, hígida, procurou atendimento com o desejo de realizar cirurgia refrativa. Nos exames pré-operatórios, a paciente apresentava acuidade visual sem correção de 20/70 no olho direito (OD) e 20/60 no olho esquerdo (OE). Com correção, apresentava acuidade visual de 20/20 em ambos os olhos (AO), não tendo sido usuária prévia de 
lentes de contato. A refração dinâmica mostrou -1,50 dioptrias (D) no OD e -1,25 D no OE. Paquimetria central foi realizada, apresentando $553 \mu \mathrm{m}$ no OD e $560 \mu \mathrm{m}$ no OE. A ceratometria não apresentou anormalidades (41,82/42,77 D no OD e 41,12/42,44 D no $\mathrm{OE}$ ), assim como a pressão intra-ocular (15 mmHg em AO). Foi realizado LASIK bilateral simultâneo, com o excimer laser LadarVision $4000^{\mathrm{TM}}$, microcerátomo $\mathrm{SKBM}^{\mathrm{TM}}$, e o procedimento transcorreu sem acidentes pré-operatórios. No primeiro dia pósoperatório, a paciente apresentou acuidade visual não corrigida de 20/40 parcial no OD e 20/60 no OE, com biomicroscopia sem alteração. A conduta nesse momento foi a utilização de Tobra$\operatorname{dex}^{\circledR}$ (tobramicina e dexametasona) quatro vezes ao dia. No oitavo dia pós-operatório, a acuidade visual sem correção era 20/50 no OD e 20/40 no OE, e a refração dinâmica foi idêntica à refração estática pré-operatória, apresentando -1,75 D no OD e -1,25 D no OE. A biomicroscopia mantinha-se sem alteração, e a conduta foi manter o Tobradex ${ }^{\circledR}$. Com 14 dias de pós-operatório, o exame permanecia igual ao da semana anterior. A biomicroscopia continuava sem alterações, mostrando-se ao exame, córnea transparente, sem edema, opacificação de interface ou alterações fundoscópicas. A conduta foi manter a corticoterapia, reduzindo para uma gota por dia a cada sete dias. Nova consulta foi realizada 45 dias após a cirurgia, e a paciente permanecia com exames inalterados. Resolvemos então medir a PIO com o tonômetro de Goldman, a qual se apresentou elevada em ambos os olhos (44 mmHg em AO). Com esse achado, suspendemos o uso do corticosteróide e iniciamos o uso de Iopidine $^{\circledR}$ (cloridrato de apraclonidina). Após dois meses de pós-operatório, a paciente apresentava acuidade visual sem correção de 20/50 parcial no OD e 20/30 no OE, sem aumento da escavação papilar, PIO de $18 \mathrm{mmHg}$ no OD e de $17 \mathrm{mmHg}$ no OE, refração dinâmica de -1,00 D em AO. Após 90 dias de pósoperatório, apresentava curva tensional diária dentro dos limites da normalidade, $\mathrm{PIO}$ de $14 \mathrm{mmHg}$ em AO, com refração plana e acuidade visual não corrigida de 20/20 em AO e ceratometria de 41,05/42,45 D no OD e 41,20/42,50 D no OE. O campo visual manteve-se normal.

\section{DISCUSSÃO}

Sabe-se que aproximadamente um terço da população pode desenvolver hipertensão ocular após o uso de corticosteróide tópico. Entretanto, não há relato na literatura de associação do seu uso com regressão miópica pós-LASIK. Antes de firmar o diagnóstico de hipertensão ocular foram descartadas as hipóteses de patologias corneanas como: ceratite lamelar difusa e ectasia ${ }^{(1-2)}$

A ceratite lamelar difusa foi descartada, pois o exame de biomicroscopia apresentou córnea transparente, sem edema ou opacificação de interface. A literatura relata que a ceratite lamelar difusa é uma reação inflamatória de etiologia desconhecida que ocorre após cirurgia refrativa. Geralmente ocorre nos primeiros dias de pós-operatório, podendo ser assintomática, e em casos mais severos apresentar sintomas de dor, visão borra- da, fotofobia e diminuição permanente de acuidade visual. Os achados encontrados na biomicroscopia são de infiltração celular na interface, podendo atingir o estroma corneano anterior ${ }^{(4)}$.

A hipótese de ectasia corneana foi descartada, pois a topografia corneana realizada no pós-operatório não apresentou critérios para sua suspeita. Além disso, a literatura mostra que a ectasia corneana manifesta-se no mínimo um ano após a cirurgia. Ectasia corneana pós-LASIK representa alteração progressiva da integridade da córnea, modificando-se para formato cônico irregular. Acarreta aumento do astigmatismo, da miopia, com, conseqüente diminuição da acuidade visual. Geralmente ocorre em córneas que apresentam espessura corneana residual pós-ablação inferior a $250 \mu \mathrm{m}^{(5-8)}$.

É importante lembrar que as alterações nos valores da PIO pós-cirurgia refrativa podem ser influenciadas pela modificação da espessura corneana(4). Isso ocorre principalmente quando são utilizados os tonômetros de aplanação e de nãocontato, pois esses apresentam medidas precisas somente quando a espessura corneana é próxima de $0,52 \mathrm{~mm}^{(5-6)}$. Córneas espessas podem resultar em medidas falsamente aumentadas, e córneas delgadas em medidas falsamente diminuídas. Isto é mais importante no caso de pressões limítrofes ${ }^{(9-11)}$.

Após o diagnóstico de hipertensão ocular severa $(44 \mathrm{mmHg}$ AO), foi suspenso o corticosteróide e iniciado o uso de Iopidine $^{\circledR}$. Com o uso de Iopidine ${ }^{\circledR}$ por 45 dias, a paciente apresentava acuidade visual não corrigida de 20/20 em AO. Acreditamos que o aumento severo da pressão intra-ocular somada a uma diminuição na espessura corneana pela ablação, tenha modificado a sua curvatura, alterando seu poder de refração.

\section{CONCLUSÃO}

A medida da pressão intra-ocular deve ser sempre realizada no pré-operatório e pós-operatório de cirurgias refrativas. O uso de corticosteróide aumenta a chance de desenvolvimento de hipertensão ocular transitória e até mesmo de glaucoma de ângulo aberto. O presente relato sugere que a hipertensão ocular possa ser um diagnóstico diferencial para diminuição da acuidade visual e regressão miópica, quando não há evidências de patologias que envolvam a córnea no pósoperatório recente de LASIK.

\section{ABSTRACT}

Corticosteroids can increase intraocular pressure when administered topically, systemically and even when inhaled. They are routinely used after refractive surgeries to lower or prevent an inflammatory action. In this case history, we present a 36year-old patient who had a total myopic regression two weeks after LASIK for low myopia, caused by steroid-induced ocular hypertension. The purpose of this report is to describe how the case was managed, the diagnostic hypothesis, and to stress the importance of intraocular pressure measurement after LASIK. 
Keywords: Myopia/surgery; Adrenal cortex hormones/adverse effects; Keratomileusis, laser in situ; Intraocular pressure; Glaucoma; Postoperative period; Case reports [Publication type]

\section{REFERÊNCIAS}

1. Hamilton DR, Manche EE, Rich LF, Maloney RK. Steroid-induced glaucoma after laser in situ keratomileusis associated with interface fluid. Ophthalmology. 2002;109(4):659-65.

2. Park JJ, Gole GA. Corticosteroid-induced glaucoma in child after a scleral reinforcement procedure. Clin Experiment Ophthalmol. 2002;30(5):372-4.

3. Kim YY, Glover BK, Shin DH, Lee D, Frenkel RE, Abreu MM. Effect of topical anti-inflammatory treatment on the long-term outcome of laser trabeculoplasty. Fluorometholone - Laser Trabeculoplasty Stud Group. Am J Ophthalmol. 1998; 126(5):721-3.

4. Bühren J, Baumeister M, Cichocki M, Kohnen T. Confocal microscopic characteristics of stage 1 to 4 diffuse lamellar keratitis after laser in situ keratomileusis. J Cataract Refract Surg. 2002;28(8):1390-9.

5. Pallikaris IG, Kymionis GD, Astyrakakis NI. Corneal ectasia induced by laser in situ keratomileusis. J Cataract Refract Surg. 2001;27(11):1796-802.

6. Argento C, Cosentino MJ, Tytiun A, Rapetti G, Zarate J. Corneal ectasia after laser in situ keratomileusis. J Cataract Refract Surg. 2001;27(9):1440-8.

7. Lafond G, Bazin R, Lajoie C. Bilateral severe keratoconus after laser in situ keratomileusis in a patient with forme fruste keratoconus. J Cataract Refract Surg. 2001;27(7):1115-8.

8. Randleman JB, Russell B, Ward MA, Thompson KP, Stulting RD. Risk factors and prognosis for corneal ectasia after LASIK. Ophthalmology. 2003; 110(2):267-75.

9. Moura CR, Paranhos Júnior A, Grottone GT, Almeida PB, Mello PAA, Prata Júnior JA. Análise da pressão intra-ocular após ceratectomia fotorrefrativa com excimer laser. Arq Bras Oftalmol. 2000;63(2):107-10.

10. Sakata K, Selonke I, Schmitt A, Garndinetti A, Sakata V, Guerra DR, Sakata L. Glaucoma de pressão normal e espessura corneana central. Arq Bras Oftalmol. 2002;65(6):619-22.

11. Meirelles SHS, Stohler NSF, Mariano MP, Yamane R, Yamane Y. Espessura corneana no glaucoma de pressão normal. Rev Bras Oftalmol. 2001;60(1):56-64. 